\title{
Predictive Factors and Rates of Fusion in Minimally Invasive Transforaminal Lumbar Interbody Fusion Utilizing rhBMP-2 or Mesenchymal Stem Cells
}

\author{
SAMUEL C. OVERLEY, MD,${ }^{1}$ STEVEN J. MCANANY, MD, ${ }^{2}$ MUHAMMAD A. ANWAR, MBBS, ${ }^{1}$ ROBERT \\ K. MERRILL, BS, ${ }^{1}$ ANDREW LOVY, MD,${ }^{1}$ JAVIER Z. GUZMAN, MD, ${ }^{1}$ SERGEY ZHADANOV, MD, ${ }^{1}$ \\ AMISH DOSHI, MD ${ }^{1}$ EDWARD ROTHENBERG, BS, ${ }^{1}$ AVANI VAISHNAV, MBBS,${ }^{2}$ CATHERINE GANG, \\ $\mathrm{MPH},{ }^{2}$ SHEERAZ A. QURESHI, MD, MBA ${ }^{2}$ \\ ${ }^{I}$ Department of Orthopedic Surgery, Icahn School of Medicine at Mount Sinai, New York, New York, ${ }^{2}$ Department of Orthopedic Surgery, Hospital for Special \\ Surgery, New York, New York
}

\begin{abstract}
Background: Several fusion adjuncts exist to enhance fusion rates during minimally invasive transforaminal lumbar interbody fusion (MI-TLIF). The objective of this study was to compare fusion rates in patients undergoing MITLIF with either rhBMP-2 or cellularized bone matrix (CBM).

Methods: We conducted a single surgeon retrospective cohort study of patients who underwent MI-TLIF with either rhBMP-2 or CBM placed in an interbody cage. Single and multilevel procedures were included. Fusion was assessed on computed tomography scans at 12-month follow-up by an independent, blinded, board-certified neuroradiologist. Fusion rates and rate of revision surgery were compared with a Fisher exact test between the 2 groups. A multivariate regression analysis was performed to identify patient factors that were predictive of radiographic nonunion after MI-TLIF.

Results: A total of 93 fusion levels in 78 patients were reviewed. Thirty-nine patients received CBM, and 39 patients received rhBMP-2. The patients receiving rhBMP-2 were older on average (61.4 vs 55.6, $P=.03$ ). The overall fusion rate was $68 \%$ in the CBM group (32/47 levels) and $78 \%$ in the rhBMP-2 group $(36 / 46)(P=.35)$. Only preoperative hypertension was predictive of radiographic nonunion (odds ratio $=3.5, P=.05$ ). There were 3 smokers in the CBM group and 4 smokers in the BMP group, and 1 in each group experienced radiographic pseudarthrosis. A total of 4 patients, 3 in the CBM group and 1 in the BMP group $(P=.61)$, required revision for symptomatic pseudarthrosis. All of these patients had a single-level index procedure.

Conclusions: There were no differences in radiographic fusion and rate of revision surgery in patients who underwent MI-TLIF with either rhBMP-2 or CBM as fusion adjuncts.

Level of Evidence: 3

Clinical Relevance: Both rhBMP-2 and CBMs can be used as effective fusion adjuncts without any clear advantage of one over the other.

Minimally Invasive Surgery

Keywords: minimally invasive transforaminal lumbar interbody fusion, bone morphogenetic protein, cellularized bone matrix, stem cells, lumbar spine fusion, rhBMP-2, TLIF
\end{abstract}

\section{INTRODUCTION}

Minimally invasive transforaminal lumbar interbody fusion (MI-TLIF) is a well-accepted surgical treatment option for patients with degenerative conditions of the lumbar spine. ${ }^{1-6}$ While several studies have reported on the short-term advantages of minimally invasive techniques, proponents of open surgery cite poor fusion success as a major deterrent to widespread adoption. ${ }^{7-12}$ In an effort to improve fusion in the interbody space in MI-TLIF, surgeons have employed a variety of fusion substrates. The rates of successful fusion in MI-TLIF have varied, with most reports relying on surgeon interpretation of plain radiographs to determine fusion success.

In an effort to improve fusion rates in spine surgery, on- and off-label use of recombinant human bone morphogenetic protein-2 (rhBMP-2) has become widespread. Although rhBMP-2 has improved fusion rates across the realm of spinal fusion procedures, concerns of side effects of 


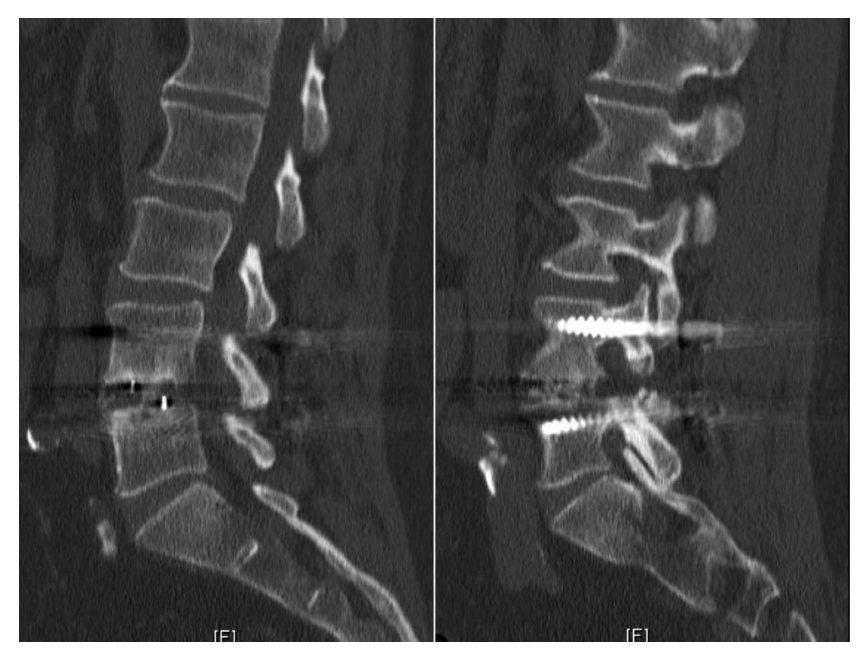

Figure 1. Representative 1-mm sagittal computed tomography cuts showing bridging bony trabeculae both within the cage and lateral to the cage. This is indicative of a solid arthrodesis.

rhBMP2 have led to bioengineering efforts to produce efficacious alternatives, especially in transforaminal interbody fusion. ${ }^{13-16}$ Specifically, in MITLIF, the literature lacks direct comparison of rhBMP-2 over other fusion substrates.

Mesenchymal stem cell containing cellular bone matrices (CBMs) were recently introduced as an alternative to rhBMP-2. CBMs offer some theoretical promise by combining osteogenic, osteoconductive, and osteoinductive characteristics that mimic the biologic profile of iliac crest bone graft. In vitro studies have demonstrated that CBMs contribute to repair and regeneration of bones, though clinical trials validating this capability in the human intervertebral disc space are sparse. ${ }^{17-19}$ Trinity Evolution is a CBM comprised of a cancellous bone matrix containing viable osteoprogenitor stem cells derived from cadaveric donor tissues. It has proven to be a safe and efficacious substrate for bone grafting procedures in some orthopedic procedures outside of the spine, though only industry-sponsored studies currently exist detailing its use and efficacy in spinal fusion procedures. ${ }^{20-23}$

The purpose of this study was to directly compare fusion success and complications in patients undergoing MI-TLIF surgery with use of rhBMP-2 (Infuse) or CBM (Trinity Evolution) in a single surgeon series. Independent, blinded neuroradiologist review of $\mathrm{CT}$ scans to assess fusion was employed, and predictive factors for nonunion were identified. To our knowledge, this study is the first of its kind to directly compare rhBMP-2 versus CBM as fusion adjuncts in MI-TLIF.

\section{METHODS}

The institutional review board approved this retrospective review of a single surgeon's patients at 1 institution between July 2011 and December 2014. Patients included in this study underwent an MI-TLIF with either rhBMP-2 (Infuse) or CBM (Trinity Elite) used as the primary fusion substrate in the interbody space. Initial cases were all treated with CBM until a specific point in time after which the remaining cases received rhBMP-2. The electronic patient medical records and the paper office charts were queried for data collection.

All patients included in this study underwent an appropriate course of nonoperative treatment, including but not limited to activity modification, physical therapy, nonsteroidal anti-inflammatory drugs, limited opioid analgesics, or epidural steroid injections, for at least 3 months. Operative indications included degenerative spondylosis or spondyolisthesis resulting in central, lateral recess or foraminal stenosis, radiculopathy, or neurogenic claudication as well as failure of nonoperative care.

Postoperative 1-mm-cut computed tomography (CT) scans were obtained 1 year after surgery for all patients in the study to assess fusion status. Two independent, blinded, board-certified neuroradiologists assessed fusion at the operative level(s). Fusion was defined as evidence of bony bridging from end plate to end plate within the cage as well as bony bridging lateral to the cage. A representative $\mathrm{CT}$ cut of a fused level is shown in Figure 1, while a case of pseudarthrosis is depicted in Figure 2.

IBM SPSS Statistics (Armonk, New York) was utilized for statistical analysis. Continuous variables were compared between cohorts with independentsample $t$ tests, and categorical variables were compared between groups with Fisher exact test. Separate analyses were performed for single-level fusions. A multivariate regression analysis was performed to identify patient factors that were predictive of radiographic nonunion after MI-TLIF. All 93 levels were individually entered into the regression model.

\section{Operative Procedure}

MI-TLIF was performed in all cases in equivalent fashion. Patients were placed prone on a Jackson table with Wilson frame. Neuromonitoring was used in all cases. Guide wires were placed into the pedicles bilaterally under AP and lateral fluorosco- 


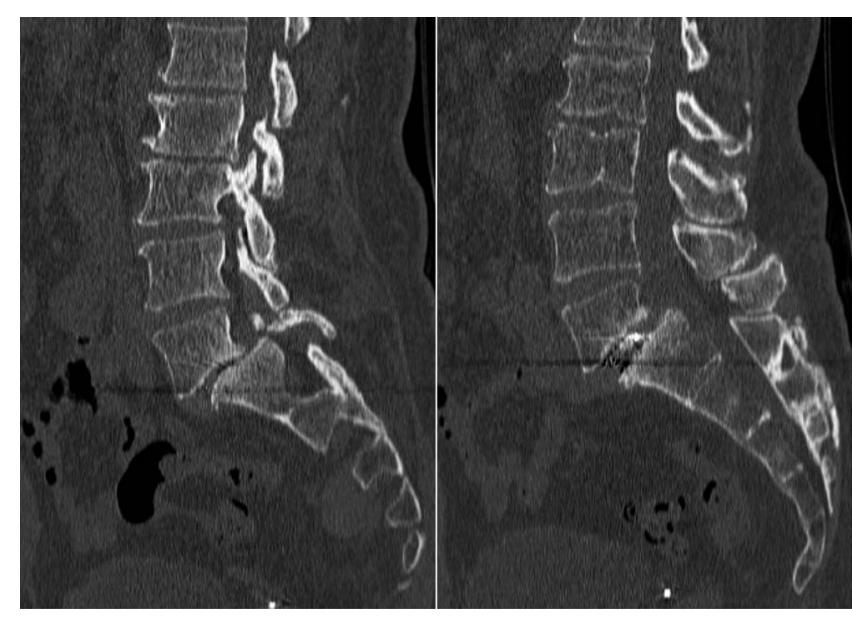

Figure 2. Representative 1-mm sagittal computed tomography cuts showing lack of bridging bony trabeculae. There is no bridging bone through or outside of the interbody cage indicative of pseudarthrosis.

py. A tubular retractor was then placed over the facet joint on the side of most significant symptoms. A complete facetectomy was performed. Thorough discectomy and end plate preparation was performed using a combination of distractors, shavers, and curettes; $10 \mathrm{~cm}^{3}$ of morecellized allograft bone was placed anteriorly in the disc space. This was followed by placement of an appropriately sized polyetheretherketone (PEEK) interbody cage. The cage was packed either with an x-small rhBMP-2 sponge or with $5 \mathrm{~cm}^{3}$ of Trinity Evolution CBM. In the rhBMP-2 group, another $5 \mathrm{~cm}^{3}$ of morcellized allograft bone was packed behind the cage in the interbody space in attempts to mitigate direct exposure of the neural elements to the rhBMP-2. In the Trinity Evolution CBM group, the remainder of the $5 \mathrm{~cm}^{3}$ of CBM not permitted in the PEEK cage was combined with $5 \mathrm{~cm}^{3}$ of morcellized allograft bone and placed posterior to the interbody cage. Cannulated pedicle screw instrumentation was performed over the previously placed guide wires. Rods and endcaps were inserted and a standard closure was performed. No patients had an additional posterolateral fusion performed.

\section{RESULTS \\ Patient Characteristics}

A total of 93 fusion levels in 78 patients were reviewed. Thirty-nine patients received Trinity Elite CBM in the interbody cage; 39 patients received Infuse rhBMP-2 in the interbody cage. The epidemiological variables for all cases as well as singlelevel-only procedures are summarized in Tables 1
Table 1. Patient demographic and operative information for all cases.

\begin{tabular}{lccc}
\hline & $\begin{array}{c}\text { Trinity } \\
\text { (CBM) } \\
\text { Variables }\end{array}$ & $\begin{array}{c}\text { rhBMP-2 } \\
(\mathbf{n}=\mathbf{3 9})\end{array}$ & $\boldsymbol{P}$ Value \\
\hline Age (average) & $55.6 \pm 11.7$ & $61.4 \pm 11.1$ & $.0271^{*}$ \\
BMI (average) & $28.0 \pm 7.4$ & $27.4 \pm 5.7$ & .6603 \\
Total levels & 47 & 46 & \\
$\quad$ Multilevel procedure & 7 & 7 & 1.0000 \\
L1-2 & 0 & 0 & 1.0000 \\
L2-3 & 0 & 0 & 1.0000 \\
L3-4 & 2 & 4 & .4349 \\
L4-5 & 23 & 28 & .2996 \\
L5-S1 & 22 & 14 & .1370 \\
Male & 17 & 24 & .1733 \\
Female & 22 & 15 & \\
Private insurance & 22 & 25 & .6439 \\
Average follow-up (mo) & $18.4 \pm 7.9$ & $13.7 \pm 4.1$ & $.0015^{*}$ \\
Smoker & 3 & 4 & 1.0000 \\
Hypertension & 21 & 17 & .4971 \\
Diabetes & 5 & 8 & .5448 \\
Levels fused & $32 / 47$ & $36 / 46$ & .3506 \\
Reoperation for pseudo & $3 / 39$ & $1 / 39$ & .6151 \\
\hline
\end{tabular}

Abbreviations: CBM, cellular bone matrix; rhBMP-2, recombinant human bone morphogenetic protein-2; BMI, body mass index.

*Statistically significant value at a threshold of $P<.05$.

and 2. The average age in the rhBMP-2 cohort was 61.4 years as compared with 55.6 in the Trinity Elite cohort $(P=.03)$. There were no statistical differences between the 2 groups with respect to BMI, insurance status, number of operative levels, smoking status, or associated medical comorbidities.

\section{Fusion Rates With Either rhBMP-2 or CBM}

The fusion rate assessed at 1 year with $\mathrm{CT}$ scan was found to be $68 \%$ in the CBM group (32/47 levels) and $78 \%$ in the rhBMP-2 group (36/46 levels). The rate of fusion was not found to be statistically significant between the 2 groups $(P=$ .35) (Table 1). When examining single-level cases only, the fusion rates for the CBM and rhBMP-2 groups were 59\% (19/32) and 78\% (25/32), respectively. Although trending toward significance in favor of BMP, these results failed to demonstrate statistical difference $(P=.18)$.

A total of 4 patients $(5 \%)$ required a revision surgery for symptomatic pseudarthrosis, 3 in the CBM group and 1 in the rhBMP-2 group $(P=.61)$. All of these patients had a single-level index procedure.

\section{Risk Factors for Nonunion}

A multivariate analysis was performed to identify the factors that were predictive of progressing to radiographic nonunion. Basic descriptive statistics and regression coefficients for all cases are shown in Table 3. The results of the regression analysis 
Table 2. Patient demographic and operative information for single-level procedures only.

\begin{tabular}{lccc}
\hline Variables & $\begin{array}{c}\text { Trinity } \\
(\mathbf{C B M}) \\
(\mathbf{n}=\mathbf{3 2})\end{array}$ & $\begin{array}{c}\text { rhBMP-2 } \\
(\mathbf{n}=\mathbf{3 2})\end{array}$ & $\boldsymbol{P}$ Value \\
\hline Age (average) & $54.7 \pm 12.3$ & $60.6 \pm 11.9$ & .0553 \\
BMI (average) & $27.8 \pm 7.5$ & $27.3 \pm 6.0$ & .7584 \\
L1-2 & 0 & 0 & 1.0000 \\
L2-3 & 0 & 0 & 1.0000 \\
L3-4 & 0 & 2 & .4921 \\
L4-5 & 16 & 21 & .3114 \\
L5-S1 & 16 & 9 & .1235 \\
Male & 13 & 21 & .0787 \\
Female & 19 & 11 & \\
Private insurance & 15 & 19 & .4527 \\
Average follow-up (mo) & $18.5 \pm 5.8$ & $14.0 \pm 4.5$ & $.0010^{\mathrm{a}}$ \\
Smoker & 2 & 3 & 1.0000 \\
Hypertension & 15 & 12 & .6131 \\
Diabetes & 4 & 6 & .7323 \\
Levels fused & $19 / 32$ & $25 / 32$ & .1769 \\
Reoperation for pseudo & $3 / 32$ & $1 / 32$ & .6128 \\
\hline
\end{tabular}

Abbreviations: CBM, cellular bone matrix; rhBMP-2, recombinant human bone morphogenetic protein-2; BMI, body mass index.

*Statistically significant value at a threshold of $P<.05$.

indicated that only preoperative hypertension was predictive of developing a radiographic pseudarthrosis (odds ration $=3.5, P=.05$ ).

\section{Complications}

Of the 78 cases performed, a total of 16 complications $(20 \%)$ occurred. The complications included 1 adjacent-level herniation, 4 pseudarthroses that required a return to the operating room, 2 cases of adjacent segment disease, 6 cases of recurrent stenosis, and 3 failures of hardware. There was no statistically significant difference in the proportion of complications between the CBM group (9/39) and the BMP group (7/39) $(P=.78)$.

\section{DISCUSSION}

MI-TLIF has been validated in the literature as a safe and effective alternative to open TLIF as well as traditional instrumented posterolateral lumbar fusion with equal fusion rates and patient-reported outcome measures. ${ }^{7-12}$ However, to date, no study has directly compared rhBMP-2 and CBM as fusion adjuncts and their effect on MI-TLIF fusion rates. Furthermore, the vast majority of relevant studies do not employ the use of stringent CT-based radiologic criteria performed by independent, board-certified, blinded neuroradiologists. This introduces the possibility of considerable observer bias.

The results of our study found utilization of rhBMP-2 to trend toward improved fusion rates in
Table 3. Regression analysis for predictors of developing radiographic nonunion.

\begin{tabular}{lcccr}
\hline Variable & $\begin{array}{c}\text { Odds } \\
\text { Ratio }\end{array}$ & $\boldsymbol{P}$ Value & $\begin{array}{c}\text { Lower } \\
\mathbf{9 5 \%} \text { CI }\end{array}$ & $\begin{array}{c}\text { Upper } \\
\mathbf{9 5 \%} \text { CI }\end{array}$ \\
\hline Age & 1.002 & .925 & 0.953 & 1.054 \\
BMI & 0.923 & .072 & 0.847 & 1.007 \\
Multilevel procedure & 0.313 & .083 & 0.084 & 1.166 \\
Private insurance & 0.790 & .699 & 0.238 & 2.619 \\
CBM & 1.581 & .401 & 0.543 & 4.598 \\
Smoking & 0.878 & .892 & 1.34 & 5.754 \\
Hypertension & 3.495 & $.050^{\mathrm{a}}$ & 1.001 & 12.198 \\
Diabetes & 0.738 & .696 & 0.161 & 3.384 \\
\hline
\end{tabular}

Abbreviations: BMI, body mass index; CBM, cellular bone matrix.

*Statistically significant value at a threshold of $P<.05$.

single- and multilevel MI-TLIF as compared to CBM, though results failed to reach statistical significance. Independent studies evaluating fusion rates after MI-TLIF with rhBMP-2 as a fusion enhancer demonstrate fusion rates ranging from $67 \%$ to $100 \%$. $^{12}$ However, there exists significant interstudy heterogeneity with different applications of type of interbody device, addition of posterolateral fusion techniques, and unilateral versus bilateral instrumentation. Villavecencio et $\mathrm{al}^{10}$ were one of the first groups to publish on fusion rates of MITLIF with rhBMP-2 without posterolateral fusion, achieving successful arthrodesis in $67 \%$ of patients. They were the only group to objectively utilize radiologist review of dynamic radiographs, though they did not employ CT. Anand et $\mathrm{al}^{24}$ published on a cohort of 100 patients who underwent MI-TLIF with rhBMP-2 as a fusion adjunct and achieved a solid fusion on $99 \%$ of their subjects. However, their fusion designation was based primarily on dynamic plain radiographs, reserving CT only for equivocal interpretations, though the literature favors CT analysis of fusion against plain radiographs. ${ }^{5}$ Additionally, fusion assessment was performed by the operating surgeon.

While rhBMP-2 is a validated fusion enhancer, the use of CBMs clinically for fusion application, though extremely prevalent in clinical practice, is sparse in the literature. Paradoxically, Grabowski et $\mathrm{al}^{25}$ estimated that CBMs were used in almost $1 / 5$ of all spinal fusion procedures performed in 2012. Currently, only 3 published studies exist evaluating the use of CBMs for spinal arthrodesis procedures. $^{26-28}$ Of these 3 studies, 2 did not disclose conflicts of interest, ${ }^{26,27}$ and the remaining study was industry sponsored. ${ }^{28}$ Fusion rates in these studies ranged from $90 \%$ to $92 \%$, paralleling previously published MI-TLIF fusion data trends. However, these studies employed only plain radiog- 
raphy and relied on surgeon interpretation of fusion status, which introduces considerable observer bias.

In our study, 2 blinded, fellowship-trained neuroradiologists assessed fusion by CT scan. Fusion, if present, was given a grade ranging from I to IV, in accordance with the spinal fusion classification system proposed by Shah et al. ${ }^{29}$ For purposes of statisitical analysis, only Shah grades III and IV (bony bridging from end plate to end plate both within and around the cage) were considered fused. ${ }^{29}$ Of the 47 levels evaluated using CBM as a fusion adjunct, $32(68 \%)$ were designated as fused, while $36 / 46(78 \%)$ levels that employed rhBMP-2 achieved solid arthrodesis. One may hypothesize that these comparatively low rates of arthrodesis can, at least in part, be attributed to the assimilation of surgeon-assessed fusion assignments in the surgical community and existing literature. When comparing our rates of fusion to those in other studies, the definition of fusion must be considered. For example, the only other study that employed the use of blinded radiologists using flexion/extension radiographs reported a $67 \%$ fusion rate for 76 single-level MI-TLIF. ${ }^{10}$

Despite a nonunion rate of $27 \%$ for all 93 levels as measured on $\mathrm{CT}$ scan, only 4 patients $(5 \%)$ required revision surgery for symptomatic pseudarthrosis, which is similar to reported rates in the literature. These findings suggest that radiographic nonunion may not prognosticate poor outcomes or the development of painful nonunion. It may be more important for the surgeon to consider revision surgery based on patient symptomatology rather than radiographic pseudarthrosis alone, as a radiographic pseudarthrosis, when independently identified by a neuroradiologist, may not correlate to a clinical, symptomatic pseudoarthrosis.

Our regression analysis revealed only preoperative hypertension to be predictive of pseudarthrosis. While an increased number of comorbidities, including hypertension, has been previously shown to predict lumbar pseudarthosis, to our knowledge, hypertension alone has never been shown to be a single predictor for nonunion. ${ }^{30,31}$ Other patientspecific variables previously implicated in poor radiological outcomes, such as smoking, diabetes, and obesity, were not found to predict poor fusion status.

Limitations of our study include the retrospective study design as well as lack of patient-reported outcome measures. While patient-reported out- comes would have been helpful to differentiate radiographic pseudarthrosis from clinical, symptomatic pseudarthrosis, the primary purpose of this article was to compare radiographic fusion between 2 fusion substrates. Thus, the lack of patientreported outcomes does not take away from the objective of this study. Additionally, the end-point time of the study, 12 months, for fusion designation may have contributed to underreporting of fusion rates in those patients who require $>12$ months for fusion maturation. Finally, our patient sample was not randomized, which may subject certain outcomes, such as fusion rates and complications, to the effect of learning the procedure, which would bias the results. However, this study was conducted when the operating surgeon was mature in the learning curve. Hence, any bias due to the learning curve would have been minimal.

\section{CONCLUSIONS}

MI-TLIF is a proven safe and efficacious means of indirectly decompressing lumbar nerve roots, restoring lordosis, and achieving a solid fusion. We found no significant difference in fusion rates between cellularized bone matrix and recombinant human bone morphogenetic protein in MI-TLIF procedures. We found hypertension to be a statistically significant risk factor for nonunion. Our stringent definition of fusion that is entirely CT scan based and requires multiple sites of bridging boney trabeculae, both within and outside of the interbody cage, as well as the employment of blinded neuroradiologists to make this designation, may suggest the possibility that previous studies have overestimated fusion rates. Further longitudinal blinded studies utilizing a standard metric for fusion as designated by third-party neuroradiologists directly comparing fusion enhancers in MITLIF procedures are needed to validate these claims.

\section{REFERENCES}

1. Adogwa O, Parker SL, Davis BJ, et al. Cost-effectiveness of transforaminal lumbar interbody fusion for Grade I degenerative spondylolisthesis. $J$ Neurosurg Spine. 2011;15(2):138-143.

2. Crandall DG, Revella J, Patterson J, Huish E, Chang M, McLemore R. Transforaminal lumbar interbody fusion with rhBMP-2 in spinal deformity, spondylolisthesis, and degenerative disease - part 2: BMP dosage-related complications and long-term outcomes in 509 patients. Spine (Phila Pa 1976). 2013;38(13):1137-1145. 
3. Crandall DG, Revella J, Patterson J, Huish E, Chang M, McLemore R. Transforaminal lumbar interbody fusion with rhBMP-2 in spinal deformity, spondylolisthesis, and degenerative disease - part 1: large series diagnosis related outcomes and complications with 2 to 9 year follow up. Spine (Phila Pa 1976). 2013; 38(13):1128-1136.

4. Yan DL, Pei FX, Li J, Soo CL. Comparative study of PILF and TLIF treatment in adult degenerative spondylolisthesis. Eur Spine J. 2008;17(10):1311-1316.

5. Williams AL, Gornet MF, Burkus JK. CT evaluation of lumbar interbody fusion: current concepts. Am J Neuroradiol. 2005;26(8):2057-2066.

6. Moskowitz A. Transforaminal lumbar interbody fusion. Orthop Clin North Am. 2002;33(2):359-366.

7. Rodríguez-Vela J, Lobo-Escolar A, Joven E, MunozMarin J, Herrera A, Velilla J. Clinical outcomes of minimally invasive versus open approach for one-level transforaminal lumbar interbody fusion at the 3- to 4-year follow-up. Eur Spine J. 2013;22(12):2857-2863.

8. Dhall SS, Wang MY, Mummaneni PV. Clinical and radiographic comparison of mini-open transforaminal lumbar interbody fusion with open transforaminal lumbar interbody fusion in 42 patients with long-term follow-up. J Neurosurg Spine. 2009;9(6):560-565.

9. Peng CW, Yue WM, Poh SY, Yeo W, Tan SB. Clinical and radiological outcomes of minimally invasive versus open transforaminal lumbar interbody fusion. Spine (Phila Pa 1976). 2009;34(13):1385-1389.

10. Villavicencio AT, Burneikiene S, Roeca CM, Nelson EL, Mason A. Minimally invasive versus open transforaminal lumbar interbody fusion. Surg Neurol Int. 2010;1:12.

11. Schwender JD, Holly LT, Rouben DP, Foley KT. Minimally invasive transforaminal lumbar interbody fusion (TLIF): technical feasibility and initial results. J Spinal Disord Tech. 2005;18(suppl):S1-S6.

12. Wu RH, Fraser JF, Härtl R. Minimal access versus open transforaminal lumbar interbody fusion: meta-analysis of fusion rates. Spine (Phila Pa 1976). 2010;35(26):2273-2281.

13. Center for Devices and Radiological Health, Food and Drug Administration, Department of Health and Human Services. Summary of safety and effectiveness data (in fuse bone graft/LT-cage lumbar tapered fusion devices). http://www. accessdata.fda.gov/scripts/cdrh/cfdocs/cftopic/pma/pma. cfm?num=P000058. Accessed March 26, 2015.

14. Schultz DG. U.S. Food and Drug Administration Public Health Notification. Life-threatening complications associated with recombinant human bone morphogenetic protein in cervical spine fusion. https:/www.fda.gov/MedicalDevices/ safety/alertsandnotices/publichealthnotifications/ucm062000. htm. Accessed March 29, 2015.

15. Even J, Eskander M, Kang J. Bone morphogenetic protein in spine surgery: current and future uses. $J$ Am Acad Orthop Surg. 2012;20(9):547-552.

16. Singh K, Nandyala SV, Marquez-Lara A, et al. Clinical sequelae after rhBMP-2 use in a minimally invasive transforaminal lumbar interbody fusion. Spine J. 2013;13(9):11181125 .

17. Bruder SP, Kraus KH, Goldberg VM, Kadiyala S. The effect of implants loaded with autologous mesenchymal stem cells on the healing of canine segmental bone defects. $J$ Bone Joint Surg Am. 1998;80(7):985-996.
18. Kon E, Muraglia A, Corsi A, et al. Autologous bone marrow stromal cells loaded onto porous hydroxyapatite ceramic accelerate bone repair in critical size defects of sheep long bones. J Biomed Mater Res. 2000;49(3):328-337.

19. Bruder SP, Kurth AA, Shea M, Hayes WC, Jaiswal N, Kadiyala S. Bone regeneration by implantation of purified, culture-expanded human mesenchymal stem cells. J Orthop Res. 1998;16(2):155-162.

20. McAnany S, Ahn J, Elboghdady IM, et al. Mesenchymal stem cell allograft as a fusion adjunct in one and two level anterior cervical discectomy and fusion: a matched cohort analysis. Spine J. 2016;16(2):163-167.

21. Rush SM, Hamilton GA, Ackerson LM. Mesenchymal stem cell allograft in revision foot and ankle surgery: a clinical and radiographic analysis. J Foot Ankle Surg. 2009;48(2):1639.

22. Rush SM. Trinity evolution: mesenchymal stem cell allografting in foot and ankle surgery. Foot Ankle Spec. 2010;3(3):140-143.

23. Guyton GP, Miller SD. Stem cells in bone grafting: trinity allograft with stem cells and collagen/beta-tricalcium phosphate with concentrated bone marrow aspirate. Foot Ankle Clin. 2010;15(4):611-619.

24. Anand N, Hamilton JF, Perri B, Miraliakbar H, Goldstein T. Cantilever TLIF with structural allograft and RhBMP2 for correction and maintenance of segmental sagittal lordosis: long-term clinical, radiographic, and functional outcome. Spine (Phila Pa 1976). 2006;31(20):E748-E753.

25. Grabowski G, Robertson RN. Bone allograft with mesenchymal stem cells: a critical review of the literature. Hard Tissue. 2013;2:20.

26. Ammerman JM, Libricz J, Ammerman MD. The role of Osteocel Plus as fusion substrate in minimally invasive instrumented transforaminal lumbar interbody fusion. Clin Neurol Neurosurg. 2013;115(7):991-994.

27. Kerr EJ III, Jawahar A, Wooten T, Kay S, Cavanaugh DA, Nunley PD. The use of osteo- conductive stem-cells allograft in lumbar interbody fusion procedures: an alternative to recombinant human bone morphogenetic protein. J Surg Orthop Adv. 2011;20(3):193-197.

28. Tohmeh AG, Watson B, Tohmeh M, Zielinski XJ. Allograft cellular bone matrix in extreme lateral interbody fusion: preliminary radio-graphic and clinical outcomes. Sci World J. 2012;2012:263637.

29. Shah RR, Mohammed S, Saifuddin A, Taylor BA. Comparison of plain radiographs with $\mathrm{CT}$ scan to evaluate interbody fusion following the use of titanium interbody cages and transpedicular instrumentation. Eur Spine $J$. 2003;12(4):378-385.

30. Gordon L, Patil CG, Lad SP, Ho C, Tian W, Boakye M. Effects of age and comorbidities on complication rates and adverse outcomes after lumbar laminectomy in elderly patients. Spine (Phila Pa 1976). 2008;33(11):1250-1255.

31. Kim YJ, Bridwell KH, Lenke LG, et al. Pseudarthrosis in long adult spinal deformity instrumentation and fusion to the sacrum: prevalence and risk factor analysis of 144 cases. Spine (Phila Pa 1976). 2006;31(20):2329-2336.

Disclosures and COI: An institutional review board approved this study. No funding was received to conduct this study. Sheeraz Qureshi is currently 
receiving grant/research support from the Cervical Spine Research Society; consulting fees from Zimmer-Biomet, Stryker Spine, Globus Medical, Inc.; and shareholder interest in Avaz Surgical and royalties from RTI, Zimmer-Biomet, Stryker Spine.

Corresponding Author: Sheeraz A. Qureshi, MD, MBA, Hospital for Special Surgery, 535 East 70th Street, New York, NY 10021. Phone: (212) 606-1585; Email: sheerazqureshimd@gmail.com.

Published 22 February 2019

This manuscript is generously published free of charge by ISASS, the International Society for the Advancement of Spine Surgery. Copyright (C) 2019 ISASS. To see more or order reprints or permissions, see http://ijssurgery.com. 